\title{
Ocupação e neoplasias intracranianas: um estudo caso-controle na região metropolitana do Rio de Janeiro, Brasil
}

\author{
Occupation and brain tumors: a case-control study in the municipality \\ of Rio de Janeiro, Brazil
}

Camila Drumond Muzi¹, Sérgio Koifman², Gina Torres Rego Monteiro³

\begin{abstract}
Resumo
Trata-se de um estudo caso-controle de base hospitalar desenvolvido na região metropolitana do Rio de Janeiro, entre 1999 e 2002, com o objetivo de estimar a magnitude da associação entre o tempo de atividade econômica e de ocupação referida com o desenvolvimento de neoplasia intracraniana em adultos. Foram coletados dados sobre a história ocupacional de 239 casos e 267 controles. As associações entre atividades econômicas e ocupações e neoplasias cerebrais foram estimadas por regressão logística. Na análise das atividades econômicas encontrou-se razão de chance com significância estatística entre o conjunto de neoplasias intracranianas e agricultura (OR: 2,52; IC95\% 1,15-5,53), e entre meningiomas e área de transporte (OR: 3,14; IC95\% 1,08-9,19) e bens imóveis (OR: 2,45; IC95\% 1,17-5,15); e estimativa de risco não significativo para meningiomas e saúde e social (OR: 3,55; IC95\% 0,89-14,16). Já a análise por ocupação apresentou estimativa de risco com significância estatística entre neoplasias intracranianas e agricultores (OR: 2,44; IC95\% 1,14-5,18) e entre gliomas e empregados de escritório (OR: 2,33; IC95\% 1,02-5,29). Os achados corroboram a hipótese de associação entre atividades econômicas/ocupações e neoplasias intracranianas, demandando pesquisas que identifiquem exposições mais específicas.

Palavras-chave: neoplasias intracranianas; ocupações; estudos de casos e controles; exposição ocupacional.
\end{abstract}

\begin{abstract}
This is a hospital-based case-control study with the objective of estimating the magnitude of association between the economic activity duration and occupation referred to the development of intracranial neoplasms in adults. We collected data on occupational history of 239 cases and 267 controls. Associations between economic activities and occupations and brain cancers were estimated by logistic regression. In the analysis of economic activities was found odds ratio with statistical significance between the number of brain tumors and Agriculture (OR: 2.52; 95\% Cl 1.15-5.53), and between meningiomas and transport area (OR: $3.14 ; 95 \% \mathrm{Cl} 1.08-9.19)$ and real estate (OR: 2.45; 95\% Cl 1.17-5.15), and risk estimate is not significant for meningiomas and Health and Social (OR: $3.55 ; 95 \% \mathrm{Cl} 0.89-14.16)$. The analysis by occupation presented risk estimates with statistical significance between brain tumors and farmers (OR: $2.4495 \% \mathrm{Cl} 1.14-5.18$ ) and between gliomas and office employees (OR: 2.33; 95\% Cl 1.02-5.29). The findings support the hypothesis of an association between economic activities/ occupations and intracranial tumors, requiring more research to identify specific exposures.
\end{abstract}

Keywords: Neoplasms, Intracranial; occupations; case-control studies; occupational exposure.

\footnotetext{
Trabalho realizado na Escola Nacional de Saúde Pública - Fundação Oswaldo Cruz (ENSP-FIOCRUZ) - Rio de Janeiro (RJ), Brasil.

${ }^{1}$ Mestre em Ciências (Saúde Pública e Meio Ambiente) pela ENSP-FIOCRUZ; Tecnologista do Instituto Nacional de Câncer - Rio de Janeiro (RJ), Brasil. 2Pós-doutor em Medicina Preventiva pela McGill University; Pesquisador Titular do Departamento de Epidemiologia e Métodos Quantitativos em Saúde da ENSP-FIOCRUZ - Rio de Janeiro (RJ), Brasil.

32Doutora em Ciências (Saúde Pública) pela ENSP-FIOCRUZ; Pesquisadora do Departamento de Epidemiologia e Métodos Quantitativos em Saúde da ENSP-FIOCRUZ - Rio de Janeiro (RJ), Brasil.

Endereço para correspondência: Camila Drumond Muzi - Rua Professor Eurico Rabelo, 149/502 - Maracanã - CEP: 20271-150 - Rio de Janeiro (RJ), Brasil E-mail: camilamuzi@gmail.com

Fonte de financiamento: nenhuma.

Conflito de interesse: nada a declarar.
} 


\section{INTRODUÇÃO}

Os tumores primários do sistema nervoso central (SNC) representam menos de $2 \%$ das neoplasias malignas. Constituem, portanto, uma pequena fração do total de pacientes com câncer na atualidade, embora sua incidência seja crescente. A evolução clínica destes tumores pode resultar em alterações mentais e déficits neurológicos irreversíveis, além de perdas sociais tão ou mais devastadoras que outras neoplasias ${ }^{1}$.

A Agência Internacional de Pesquisa em Câncer (IARC) estimou, em 2008, a incidência de tumores cerebrais, ajustada por idade pela população mundial, de 5,8/100.000 em homens e 4,4/100.000 em mulheres, nos países mais desenvolvidos, enquanto nos menos desenvolvidos as taxas seriam de 3,2/100.000 em homens e 2,8/100.000 em mulheres. Neste relatório, o Brasil apresenta taxas de 5,3/100.000 em homens e 3,9/100.000 em mulheres ${ }^{2}$. No que diz respeito à tendência temporal, as taxas de incidência e mortalidade por neoplasias intracranianas têm aumentado no Brasil e no mundo nos últimos anos ${ }^{3,4}$.

Algumas hipóteses são apontadas na literatura para explicar o incremento nas taxas de incidência e mortalidade dos tumores do SNC. A maior deteç̧ão de casos seria fruto das revisões na Classificação Internacional de Doenças (CID) e da mudança no acesso aos serviços de saúde, que propiciariam maior facilidade para o diagnóstico da doença, especificamente quanto ao uso cada vez maior de exames de imagem, como a tomografia computadorizada e a ressonância magnética. Adicionalmente, maior atenção à saúde do idoso, tendo em vista o crescente envelhecimento populacional, também contribuiria para o aumento no diagnóstico de casos da doença ${ }^{1}$.

Entre os diversos fatores ambientais de risco para o desenvolvimento do câncer, sugere-se que os de origem ocupacional apresentem maior potencial de controle. Entretanto, apenas $20 \%$ das substâncias químicas em uso no ambiente de trabalho contam com informações toxicológicas adequadas ${ }^{5}$.

Diversos estudos epidemiológicos investigaram a associação entre ocupações ou exposições ocupacionais com tumores do SNC, entre os quais, fazendeiros e agricultores ${ }^{6,7}$, trabalhadores da indústria da borracha ${ }^{8}$ e da petroquímica ${ }^{9}$, profissionais da área de saúde ${ }^{10-12} \mathrm{e}$ trabalhadores expostos a campos eletromagnéticos de baixa frequência ${ }^{13}$.

Tendo em vista a realização de um estudo caso-controle sobre fatores de risco para tumores intracranianos em adultos no Rio de Janeiro, o presente trabalho foi desenvolvido com o objetivo analisar a história ocupacional e explorar a possível associação entre as atividades econômicas e as ocupações referidas por seus participantes e o desenvolvimento de tumores intracranianos.

\section{MÉTODOS}

\section{Antecedentes}

Durante o período entre abril de 1999 e dezembro de 2002, foi realizado na Região Metropolitana do Rio de Janeiro um estudo caso-controle de base hospitalar para explorar os fatores de risco para as neoplasias do SNC. Realizou-se o recrutamento de casos incidentes de tumores intracranianos em pacientes com idade de 30 a 65 anos. Entre eles, 80,0\% tiveram confirmação diagnóstica por histopatologia, 15,8\% apresentaram imagem radiológica sugestiva, e 4,2\% relato cirúrgico de tumor primário intracraniano. As lâminas dos casos com confirmação histopatológica foram revisadas por dois patologistas e, em caso de discordância, por um terceiro, sendo, em todas as etapas, realizada revisão às cegas do material analisado ${ }^{14}$.

Os controles foram pacientes internados majoritariamente nas mesmas instituições de identificação dos casos, pareados por frequência de idade e sexo, portadores de nosologias selecionados nos setores hospitalares de ortopedia $(47,0 \%)$, cirurgia geral $(22,0 \%)$, ginecologia $(14,2 \%)$ e neurocirurgia $(7,1 \%)$, entre outros $(9,7 \%)$, residentes na mesma região. No processo de seleção, foram excluídos os pacientes internados com história clínica de neoplasia maligna ou por sintomas potencialmente associados à clínica de tumores de cérebro (como convulsão, distúrbio neurológico, etc.) $)^{14}$.

\section{População de estudo}

Foram identificados 306 casos potencialmente elegíveis para o estudo, sendo que 17 (5,6\%) não chegaram a ser entrevistados por recusa ou falecimento. E dos 289 remanescentes, 49 (17\%) foram descartados por não cumprirem os critérios de inclusão.

Para o presente estudo, foram ainda excluídos dois indivíduos, sendo que em um caso o paciente nunca havia trabalhado e em um controle constava ocupação única com menos de seis meses de exercício. Assim, foram analisados 239 casos (85 meningiomas e 66 gliomas) e 267 controles pareados por faixa etária e sexo.

\section{História ocupacional}

As ocupações relatadas pelos participantes no estudo de 1999 a 2002 foram classificadas tendo em vista o ramo da atividade econômica pela Statistical Classification of Economics Activities in the European Community (NACE) ${ }^{15}$, que permite a comparação com dados nacionais e internacionais. Esta classificação é composta por 17 seções de atividades, subdivididas em grupos e classes que abrangem os diversos processos produtivos.

Para a classificação das ocupações, foi utilizada a International Standard Classification of Occupations (ISCO) ${ }^{16}$, da Organização Internacional do Trabalho, na qual a ocupação é o menor segmento do trabalho 
especificamente identificado no sistema de classificação, composto por nove seções. A ISCO foi utilizada para que fosse possível a comparação, a posteriori, com outros estudos internacionais que explorassem a mesma associação pretendida pelo presente artigo.

Todos os sujeitos do estudo foram codificados de acordo com ambas as classificações por um dos autores (Camila Drumond Muzi). Na análise da história ocupacional utilizou-se o teste de Mann-Whitney para a comparação do tempo médio de trabalho entre casos e controles nos diferentes grupos NACE e ISCO. Foi perguntada aos participantes do estudo toda a sua história ocupacional e realizada a análise do histórico de trabalho dos participantes para a estimativa da associação.

\section{Análise estatística}

A avaliação da presença de heterogeneidade na distribuição do tempo de trabalho exercido por casos e controles nas diferentes atividades econômicas (classificação NACE) ou atividade ocupacional (classificação ISCO) foi determinada pelo emprego do teste do $\chi^{2}$. Considerou-se a presença de heterogeneidade quando o valor $\mathrm{p}$ foi $<0,05$.

A variável idade foi estratificada em: menores de 39 anos; pessoas entre 40 e 49 anos; de 50 a 59 anos, e com 60 anos ou mais. Em relação à variável escolaridade, foram considerados dois estratos: aqueles com menos de oito anos de estudo e indivíduos com oito anos ou mais.

As estimativas de risco relativo nos diversos ramos de atividade econômica, assim como de ocupação, e os tumores intracranianos foram calculados para o conjunto de neoplasias do SNC, para gliomas e meningiomas por meio da determinação de razões de chances (Odds ratio - OR) brutas e ajustadas por sexo, faixa etária e escolaridade, analisadas pela regressão logística não condicional, e adotando-se nível de significância estatística de 95\% pelo método de Wald.

Além disso, avaliou-se a presença de gradientes de risco/proteção conforme a variação das razões de chance, segundo tempo de trabalho, utilizando-se o Teste do $\chi^{2}$ de tendência e considerando-se estatisticamente significativa quando valor $\mathrm{p}<0,05$.

Para a realização da análise estatística, foi usado o programa Statistical Package for the Social Sciences (SPSS) for Windows 13.0.

\section{Aspectos éticos}

O estudo foi submetido e aprovado pelos Comitês de Ética em Pesquisa da Escola Nacional de Saúde Pública (Parecer $n^{\circ}$ 25/08) e dos hospitais onde foi realizada a investigação.

\section{RESULTADOS}

A população do estudo foi composta por 239 casos e 267 controles, sendo que $57,7 \%$ dos casos e $60,3 \%$ dos controles eram do sexo feminino. A idade média dos casos foi 47,5 anos (desvio padrão de 9,6 anos) e a dos controles 46,6 anos (desvio padrão de 9,6 anos). A distribuição da frequência da variável escolaridade foi semelhante nos dois grupos, sendo menor que 8 anos em cerca de $70 \%$ dos participantes. Acima de $60 \%$ dos indivíduos de ambos os grupos relataram antecedentes de união conjugal e residência no município do Rio de Janeiro. As características sociodemográficas de casos e controles não apresentaram diferenças estatisticamente significativas (Tabela 1).

Quando o indivíduo selecionado não estava em condição de responder ao questionário, as informações foram obtidas de respondentes substitutos, o que ocorreu em $36(15,1 \%)$ casos e $11(4,1 \%)$ controles. O conjunto dos participantes do estudo apresentou a média de 2,28 ocupações por pessoa, sendo similar para casos e controles: 2,29 e 2,27, respectivamente. No subconjunto cuja informação foi dada por substitutos, o número médio de ocupações foi 1,81 nos casos e 1,54 nos controles.

Tabela 1. Características selecionadas de casos de tumores intracranianos e controles no Rio de Janeiro entre 1999 e 2002

\begin{tabular}{|c|c|c|c|c|c|}
\hline \multirow[t]{2}{*}{ Variáveis } & \multicolumn{2}{|c|}{$\begin{array}{c}\text { Casos } \\
(n=239)\end{array}$} & \multicolumn{2}{|c|}{$\begin{array}{c}\text { Controles } \\
(n=267)\end{array}$} & \multirow{2}{*}{$\begin{array}{c}\text { Valor } \\
\mathrm{p}\end{array}$} \\
\hline & $\mathrm{n}$ & $\%$ & $\mathbf{n}$ & $\%$ & \\
\hline \multicolumn{6}{|l|}{ Sexo } \\
\hline Masculino & 101 & 42,3 & 106 & 39,7 & \multirow[t]{2}{*}{0,56} \\
\hline Feminino & 138 & 57,7 & 161 & 60,3 & \\
\hline \multicolumn{6}{|l|}{ Idade (anos) } \\
\hline$<39$ & 55 & 23,0 & 69 & 25,8 & \multirow[t]{4}{*}{0,57} \\
\hline $40-49$ & 78 & 32,6 & 96 & 36,0 & \\
\hline $50-59$ & 71 & 29,7 & 69 & 25,8 & \\
\hline$\geq 60$ & 35 & 14,7 & 33 & 12,4 & \\
\hline \multicolumn{6}{|l|}{ Escolaridade (anos) } \\
\hline$<8$ & 167 & 69,9 & 183 & 68,5 & \multirow[t]{2}{*}{0,75} \\
\hline$>8$ & 72 & 30,1 & 84 & 31,5 & \\
\hline \multicolumn{6}{|l|}{ Estado civil } \\
\hline Solteiro & 36 & 15,1 & 34 & 12,7 & \multirow[t]{4}{*}{0,72} \\
\hline Casado & 150 & 62,7 & 174 & 65,2 & \\
\hline Divorciado & 33 & 13,8 & 32 & 12,0 & \\
\hline Viúvo & 20 & 8,4 & 27 & 10,1 & \\
\hline \multicolumn{6}{|l|}{ Cidade de residência } \\
\hline Rio de Janeiro & 154 & 64,5 & 184 & 69,0 & \multirow[t]{6}{*}{0,53} \\
\hline Duque de Caxias & 16 & 6,7 & 19 & 7,1 & \\
\hline Nova Iguaçu & 13 & 5,4 & 18 & 6,7 & \\
\hline São João de Meriti & 12 & 5,0 & 9 & 3,4 & \\
\hline Belford Roxo & 8 & 3,3 & 10 & 3,7 & \\
\hline Outra & 36 & 15,1 & 27 & 10,1 & \\
\hline
\end{tabular}




\section{Ramo de atividade econômica}

As atividades econômicas na Agricultura (NACE 1) revelaram associação positiva com as neoplasias do SNC (todos os tumores), sendo estatisticamente significativas para o estrato daqueles com tempo de trabalho igual ou superior a 6 anos (OR ajustada, OR aj: 2,52 (IC95\% 1,15-5,53)). Foi também observada a presença de efeito dose-resposta relativo ao tempo de trabalho na atividade agrícola e neoplasia do SNC (valor p de tendência: 0,013 ). A associação positiva se manteve na análise dos gliomas e meningiomas, embora apenas o primeiro tivesse apresentado tendência estatisticamente significativa em relação ao tempo de trabalho (Tabela 2).

Aqueles que relataram trabalhar nos ramos de Transportes (NACE 9) e Bens Imóveis (NACE 11), por 6 anos ou mais, apresentaram estimativas de risco elevadas para meningiomas: $\mathrm{OR}_{\mathrm{aj}}$ : 3,14 (IC95\% 1,08-9,19) e OR $\mathrm{aj}$ : 2,45 (IC95\% 1,17-5,15), respectivamente. Já no grupo dos Empregados Domésticos (NACE 16), a associação positiva não foi estatisticamente significativa em cada estrato, mas observou-se tendência a excesso de risco para meningiomas com o tempo de atividade, com valor p de 0,0273 (Tabela 2).

Os trabalhadores nos ramos da Construção (NACE 6), Hotéis (NACE 8) e Administração Pública (NACE 12) apresentaram tendência de proteção ao desenvolvimento de meningiomas em relação ao tempo de trabalho, estatisticamente significativa, com valor $\mathrm{p}$ de 0,0279, 0,0211 e 0,0275, respectivamente (Tabela 2).

\section{Ocupação}

Os trabalhadores da agricultura (ISCO 6), com 6 ou mais anos nesta atividade, apresentaram $\mathrm{OR}_{\mathrm{aj}}$ de 2,44 (IC95\% $1,14-5,18)$ para todos os tumores do SNC, verificando-se tendência estatisticamente significativa segundo tempo de trabalho (valor $\mathrm{p}$ de 0,03 ). Foi observada tendência limítrofe (valor $\mathrm{p}$ de $0,059)$ relativa à associação entre tempo de trabalho e o desenvolvimento de gliomas, e $\mathrm{OR}_{\mathrm{aj}}$ de 1,35 (IC95\% 0,97-2,38) para o conjunto de tumores do SNC nos que relataram terem sido Empregados de escritório (ISCO 3) por 6 ou mais anos. Essa associação revelou maior magnitude e significância estatística $\mathrm{OR}_{\mathrm{aj}}$ de 2,33 (IC95\% 1,02-5,29) na análise dos gliomas (Tabela 3).

O grupo de Trabalhadores com Vendas (ISCO 4) apresentou tendência protetora para gliomas $(\mathrm{p}=0,458)$ segundo o tempo nessa ocupação (Tabela 3).

\section{DISCUSSÃO}

A partir de informações coletadas entre os participantes de um estudo caso-controle de base hospitalar, o presente trabalho buscou explorar a associação entre ramo de atividade econômica e ocupação no desenvolvimento de neoplasias intracranianas em adultos da Região Metropolitana do Rio de Janeiro.

Os resultados desta análise são indicativos da presença de uma associação positiva entre a atividade ocupacional na agricultura e o desenvolvimento de tumores cerebrais, já relatada em outros estudos ${ }^{6,7,11}$. As análises da atividade econômica na Agricultura (NACE 1), assim como a da atividade ocupacional Agricultores (ISCO 6), apresentaram gradiente positivo e estatisticamente significativo nas estimativas de risco do conjunto das neoplasias intracranianas, segundo tempo de trabalho. Ao avaliar por subtipo histológico (gliomas e meningiomas), evidenciou-se associação positiva utilizando ambas as classificações, embora com tendência significativa apenas nos gliomas e na NACE.

Diversos estudos têm examinado a associação entre trabalho na agricultura (fazendeiros/agricultores) e o conjunto de tumores cerebrais, sendo, todavia, escassas as publicações que examinem esta associação segundo tipos histológicos específicos ${ }^{7,11}$.

Nesta investigação, os participantes classificados no ramo de atividade econômica Agricultura (NACE 1) incluiu pecuaristas, avicultores, trabalhadores de culturas diversas e outros. Como o estudo foi conduzido na região urbana do Rio de Janeiro, é plausível que a população analisada tenha vivido em área rural por tempo variado e, assim, estaria vulnerável a exposições peridomiciliares, como, por exemplo, aplicação de pesticidas em grandes áreas. Além disso, este estudo não permite discriminar se a associação se deve somente a exposições inerentes ao processo produtivo do plantio/colheita de alimentos, à criação de animais ou a ambos. Não obstante, este resultado pode ser analisado como uma evidência adicional para a hipótese etiológica dos tumores cerebrais estarem associados a exposições ambientais presentes no meio rural, entre as quais se encontra o uso de pesticidas em lavouras, exposição a agentes infecciosos e aplicação de inseticidas em animais.

Foi também verificada uma associação positiva, e estatisticamente significativa, entre atividade prolongada (6 ou mais anos) no ramo de Transportes (NACE 9) e o desenvolvimento de meningiomas. A seção Transportes da Classificação NACE engloba as seguintes atividades: condução de pessoas ou cargas por via terrestre, marítima e aérea; atividades de agência de viagens e assistência turística; e telecomunicações e postagem.

Pan et al. ${ }^{12}$ evidenciaram razão de chances de 1,43 (IC95\% 1,02-2,02) para o desenvolvimento de gliomas entre trabalhadores da área de transporte e operação de equipamentos. Schlehofer et al. ${ }^{17}$ relataram a mesma magnitude de associação que o estudo anterior, particularmente em gliomas de baixo grau, enquanto para os de alto grau a razão de chances foi limítrofe. Vale notar que trabalhadores desta área encontram-se usualmente expostos a derivados do petróleo 
Tabela 2. Odds ratio bruta e ajustada do período de tempo de atividade econômica (Statistical Classification of Economics Activities in the European Community) em anos para neoplasias intracranianas ( $n=239)$, gliomas $(n=66)$ e meningiomas $(n=85)$ no Rio de Janeiro entre 1999 e 2002

\begin{tabular}{|c|c|c|c|c|c|c|}
\hline \multirow[b]{2}{*}{$\begin{array}{l}\text { NACE - tempo de } \\
\text { atividade (anos) }\end{array}$} & \multicolumn{2}{|c|}{ Neoplasias intracranianas } & \multicolumn{2}{|c|}{ Gliomas } & \multicolumn{2}{|c|}{ Meningiomas } \\
\hline & $\begin{array}{l}\text { OR bruta } \\
\text { (IC95\%) }\end{array}$ & $\begin{array}{l}\text { OR ajustada* } \\
\text { (IC95\%) }\end{array}$ & $\begin{array}{l}\text { OR bruta } \\
\text { (IC95\%) }\end{array}$ & $\begin{array}{l}\text { OR ajustada* } \\
\text { (IC95\%) }\end{array}$ & $\begin{array}{l}\text { OR bruta } \\
\text { (IC95\%) }\end{array}$ & $\begin{array}{l}\text { OR ajustada } \\
\text { (IC95\%) }\end{array}$ \\
\hline \multicolumn{7}{|c|}{ NACE 1 - Agricultura } \\
\hline 0 & 1,00 & 1,00 & 1,00 & 1,00 & 1,00 & 1,00 \\
\hline $1-5$ & $1,35(0,47-3,91)$ & $1,32(0,50-3,51)$ & $2,22(0,65-7,64)$ & $2,18(0,63-7,54)$ & $1,66(0,49-5,67)$ & $1,99(0,55-7,20)$ \\
\hline$\geq 6$ & $2,63(1,16-6,11)$ & $2,52(1,15-5,53)$ & $2,67(0,93-7,65)$ & $2,54(0,87-7,44)$ & $1,99(0,70-5,66)$ & $1,84(0,62-5,46)$ \\
\hline $\mathrm{p}$ de tendência & & 0,0131 & & 0,0426 & & 0,1757 \\
\hline \multicolumn{7}{|c|}{ NACE 4 - Manufatura } \\
\hline 0 & 1,00 & 1,00 & 1,00 & 1,00 & 1,00 & 1,00 \\
\hline $1-5$ & $1,25(0,76-2,03)$ & $1,26(0,79-2,01)$ & $1,20(0,61-2,34)$ & $1,17(0,60-2,30)$ & $1,09(0,58-2,05)$ & $1,08(0,56-2,06)$ \\
\hline$\geq 6$ & $1,02(0,64-1,63)$ & $0,98(0,62-1,54)$ & $0,57(0,25-1,28)$ & $0,55(0,24-1,25)$ & $0,52(0,25-1,09)$ & $0,56(0,26-1,19)$ \\
\hline $\mathrm{p}$ de tendência & & 0,8018 & & 0,2529 & & 0,1185 \\
\hline \multicolumn{7}{|c|}{ NACE 6 - Construção } \\
\hline 0 & 1,00 & 1,00 & 1,00 & 1,00 & 1,00 & 1,00 \\
\hline $1-5$ & $1,10(0,43-2,79)$ & $0,99(0,40-2,44)$ & $1,43(0,44-4,65)$ & $1,41(0,40-4,96)$ & $0,26(0,03-2,04)$ & $0,49(0,06-4,13)$ \\
\hline$\geq 6$ & $0,79(0,40-1,56)$ & $0,69(0,35-1,37)$ & $0,49(0,14-1,69)$ & $0,44(0,21-1,62)$ & $0,34(0,10-1,16)$ & $0,52(0,14-1,92)$ \\
\hline $\mathrm{p}$ de tendência & & 0,4899 & & 0,3179 & & 0,0279 \\
\hline \multicolumn{7}{|l|}{ NACE 7 - Atacadista } \\
\hline 0 & 1,00 & 1,00 & 1,00 & 1,00 & 1,00 & 1,00 \\
\hline $1-5$ & $1,47(0,82-2,62)$ & $1,49(0,86-2,59)$ & $0,80(0,31-2,02)$ & $0,81(0,32-2,08)$ & $1,30(0,61-2,77)$ & $1,59(0,72-3,51)$ \\
\hline$\geq 6$ & $0,97(0,58-1,63)$ & $0,91(0,55-1,52)$ & $0,82(0,38-1,80)$ & $0,80(0,35-1,79)$ & $0,91(0,45-1,84)$ & $1,22(0,59-2,56)$ \\
\hline $\mathrm{p}$ de tendência & & 0,8150 & & 0,4984 & & 0,8991 \\
\hline \multicolumn{7}{|l|}{ NACE 8 - Hotéis } \\
\hline 0 & 1,00 & 1,00 & 1,00 & 1,00 & 1,00 & 1,00 \\
\hline $1-5$ & $0,56(0,29-1,07)$ & $0,58(0,31-1,08)$ & $0,55(0,21-1,47)$ & $0,56(0,21-1,52)$ & $0,44(0,17-1,17)$ & $0,48(0,18-1,28)$ \\
\hline$\geq 6$ & $0,79(0,30-2,05)$ & $0,80(0,33-1,94)$ & $0,62(0,14-2,87)$ & $0,63(0,14-2,93)$ & $0,24(0,03-1,84)$ & $0,25(0,03-1,98)$ \\
\hline p de tendência & & 0,1161 & & 0,1851 & & 0,0211 \\
\hline \multicolumn{7}{|l|}{ NACE 9 - Transporte } \\
\hline 0 & 1,00 & 1,00 & 1,00 & 1,00 & 1,00 & 1,00 \\
\hline $1-5$ & $0,47(0,14-1,46)$ & $0,47(0,16-1,38)$ & $0,75(0,16-3,49)$ & $0,83(0,17-4,06)$ & $0,25(0,03-2,03)$ & $0,56(0,07-4,66)$ \\
\hline$\geq 6$ & $1,58(0,76-3,31)$ & $1,48(0,71-3,07)$ & $1,55(0,58-4,14)$ & $1,55(0,54-4,46)$ & $1,45(0,57-3,70)$ & $3,14(1,08-9,19)$ \\
\hline p de tendência & & 0,4589 & & 0,5635 & & 0,8578 \\
\hline \multicolumn{7}{|c|}{ NACE 11 - Bens imóveis } \\
\hline 0 & 1,00 & 1,00 & 1,00 & 1,00 & 1,00 & 1,00 \\
\hline $1-5$ & $0,66(0,37-1,14)$ & $0,68(0,40-1,17)$ & $0,36(0,12-1,06)$ & $0,40(0,13-1,21)$ & $0,60(0,27-1,35)$ & $0,72(0,31-1,67)$ \\
\hline$\geq 6$ & $1,43(0,83-2,47)$ & $1,51(0,88-2,60)$ & $1,74(0,86-3,52)$ & $1,53(0,72-3,26)$ & $2,03(1,07-3,86)$ & $2,45(1,17-5,15)$ \\
\hline $\mathrm{p}$ de tendência & & 0,5097 & & 0,4751 & & 0,1328 \\
\hline \multicolumn{7}{|c|}{ NACE 12 - Administração pública } \\
\hline 0 & 1,00 & 1,00 & 1,00 & 1,00 & 1,00 & 1,00 \\
\hline $1-5$ & $1,30(0,62-2,69)$ & $1,30(0,63-2,69)$ & $1,26(0,45-3,56)$ & $1,49(0,47-4,67)$ & $0,17(0,02-1,30)$ & $0,29(0,04-2,37)$ \\
\hline$\geq 6$ & $1,51(0,60-3,79)$ & $1,48(0,61-3,60)$ & $2,14(0,70-6,52)$ & $2,54(0,75-9,63)$ & $0,29(0,04-2,29)$ & $0,43(0,05-3,67)$ \\
\hline p de tendência & & 0,2824 & & 0,2222 & & 0,0275 \\
\hline \multicolumn{7}{|l|}{ NACE 13 - Educação } \\
\hline 0 & 1,00 & 1,00 & 1,00 & 1,00 & 1,00 & 1,00 \\
\hline $1-5$ & $1,68(0,23-14,51)$ & $1,80(0,29-11,20)$ & - & - & $1,56(0,14-17,44)$ & $1,16(0,10-13,93)$ \\
\hline$\geq 6$ & $1,00(0,34-2,87)$ & $1,07(0,39-2,93)$ & $0,89(0,19-4,20)$ & $0,93(0,18-4,75)$ & $0,69(0,15-3,28)$ & $0,56(0,11-2,82)$ \\
\hline p de tendência & & 0,9849 & & 0,5875 & & 0,5939 \\
\hline \multicolumn{7}{|c|}{ NACE 14 - Saúde e social } \\
\hline 0 & 1,00 & 1,00 & 1,00 & 1,00 & 1,00 & 1,00 \\
\hline $1-5$ & $0,39(0,13-1,06)$ & $0,41(0,15-1,07)$ & $0,50(0,11-2,22)$ & $0,54(0,12-2,48)$ & $0,76(0,25-2,33)$ & $0,74(0,24-2,35)$ \\
\hline$\geq 6$ & $2,19(0,59-8,75)$ & $2,24(0,65-7,72)$ & $1,99(0,36-11,12)$ & $2,08(0,36-11,09)$ & $4,05(1,06-15,45)$ & $3,55(0,89-14,16)$ \\
\hline p de tendência & & 0,8638 & & 0,8586 & & 0,1897 \\
\hline
\end{tabular}


Tabela 2. Continuação

\begin{tabular}{|c|c|c|c|c|c|c|}
\hline \multirow[b]{2}{*}{$\begin{array}{l}\text { NACE - tempo de } \\
\text { atividade (anos) }\end{array}$} & \multicolumn{2}{|c|}{ Neoplasias intracranianas } & \multicolumn{2}{|c|}{ Gliomas } & \multicolumn{2}{|c|}{ Meningiomas } \\
\hline & $\begin{array}{l}\text { OR bruta } \\
\text { (IC95\%) }\end{array}$ & $\begin{array}{l}\text { OR ajustada* } \\
\text { (IC95\%) }\end{array}$ & $\begin{array}{l}\text { OR bruta } \\
\text { (IC95\%) }\end{array}$ & $\begin{array}{l}\text { OR ajustada* } \\
\text { (IC95\%) }\end{array}$ & $\begin{array}{l}\text { OR bruta } \\
\text { (IC95\%) }\end{array}$ & $\begin{array}{l}\text { OR ajustada } \\
\text { (IC95\%) }\end{array}$ \\
\hline \multicolumn{7}{|c|}{ NACE 15 - Outras atividades comerciais e serviços } \\
\hline 0 & 1,00 & 1,00 & 1,00 & 1,00 & 1,00 & 1,00 \\
\hline $1-5$ & $1,62(0,56-4,82)$ & $1,69(0,63-4,54)$ & $1,13(0,23-5,59)$ & $1,19(0,24-5,93)$ & $1,82(0,52-6,40)$ & $1,94(0,52-7,18)$ \\
\hline$\geq 6$ & $1,03(0,40-2,09)$ & $1,00(0,41-2,41)$ & $0,36(0,05-2,84)$ & $0,32(0,04-2,60)$ & $0,87(0,24-3,20)$ & $0,62(0,16-2,37)$ \\
\hline $\mathrm{p}$ de tendência & & 0,7363 & & 0,3005 & & 0,9806 \\
\hline \multicolumn{7}{|c|}{ NACE 16 - Empregados domésticos } \\
\hline 0 & 1,00 & 1,00 & 1,00 & 1,00 & 1,00 & 1,00 \\
\hline $1-5$ & $0,64(0,37-1,11)$ & $0,68(0,40-1,16)$ & $0,92(0,42-2,01)$ & $0,98(0,44-2,21)$ & $1,23(0,62-2,47)$ & $1,14(0,55-2,38)$ \\
\hline$\geq 6$ & $1,08(0,72-1,63)$ & $1,15(0,73-1,79)$ & $1,67(0,92-3,02)$ & $1,86(0,94-3,66)$ & $1,88(1,09-3,24)$ & $1,47(0,78-2,75)$ \\
\hline p de tendência & & 0,8634 & & 0,1202 & & 0,0273 \\
\hline \multicolumn{7}{|c|}{ Outros (NACE 3,5 e 10 ) } \\
\hline 0 & 1,00 & 1,00 & 1,00 & 1,00 & 1,00 & 1,00 \\
\hline $1-5$ & $0,27(0,01-2,58)$ & $0,21(0,02-1,93)$ & & & $0,78(0,09-7,08)$ & $0,90(0,09-9,00)$ \\
\hline$\geq 6$ & $0,31(0,04-1,64)$ & $0,28(0,06-1,40)$ & & & $0,89(0,18-4,38)$ & $1,39(0,26-7,52)$ \\
\hline $\mathrm{p}$ de tendência & & 0,0895 & & & & 0,6956 \\
\hline
\end{tabular}

*OR ajustada por sexo, faixa etária e escolaridade

NACE: Statistical Classification of Economics Activities in the European Community; OR: Odds ratio; IC95\%: Intervalo de confiança de 95\%

(como a gasolina), solventes, fumaça proveniente da queima de óleos combustíveis na exaustão de motores e chumbo ${ }^{11,17}$.

O ramo econômico de Bens imóveis (NACE 11) incorpora as atividades do setor imobiliário, locações e outros negócios relacionados com informática, processamento de dados, pesquisa científica e de marketing, recursos humanos, consultorias, secretariado, etc. No presente estudo, os trabalhadores dessa área, com tempo igual ou superior a 6 anos na atividade, apresentaram duplicação da estimativa de risco para meningiomas $\left(\mathrm{OR}_{\mathrm{aj}}: 2,45\right)$.

Uma pequena elevação na estimativa de risco para todos os tumores intracranianos foi verificada para a ocupação Empregados de Escritório (ISCO 3) com tempo igual ou superior a 6 anos na atividade $\left(\mathrm{OR}_{\mathrm{aj}}: 1,35\right)$, assim como para gliomas no mesmo estrato de tempo de trabalho $\left(\mathrm{OR}_{\mathrm{aj}}: 2,33\right)$. Vale observar que as ocupações incluídas no grupo de Empregados de Escritório (ISCO 3) estão contidas, em sua maioria, no ramo de atividade econômica Bens imóveis (NACE 11).

A associação detectada entre aqueles que desempenharam atividades no ramo de bens imóveis e o desenvolvimento de meningiomas foi similar ao descrito por Preston-Martin et al. ${ }^{18}$ que encontraram razão de chances de $2,8(\mathrm{p}=0,03)$ de meningiomas em trabalhadores com mais de 5 anos de atividade como gerentes e administradores.

Uma possível hipótese explicativa para a associação entre o trabalho na atividade de bens imóveis com os tumores do SNC seria o contato próximo com uma diversidade de pessoas, muitas vezes em ambiente fechado, possibilitando a ocorrência de infecções por vírus ${ }^{19}$. Archer et al. ${ }^{20}$ relataram a indução de gliomas de alto grau na presença de vírus com Ribonucleic acid (RNA) oncogênico, sendo, entretanto, ainda muito incipientes os estudos que apoiem esta associação.

$\mathrm{O}$ ramo de atividade econômica Administração pública (NACE 12) contempla diversas profissões de cunho burocrático e defesa, como administradores de programas socioeconômicos (governamentais ou não), área jurídica, seguradores sociais, agentes de defesa civil, policiais, militares e bombeiros.

Outro estudo realizado no Brasil, com militares da marinha, evidenciou que os tumores cerebrais eram a $5^{\text {a }}$ neoplasia mais comum na corporação, sendo a principal causa de morte por câncer, na análise de razão de mortalidade proporcional ajustada por idade $\left(\mathrm{RMPC}_{\text {ajst }}: 339,3 \%\right)$, tendo como referência a população masculina do estado do Rio de Janeiro ${ }^{21}$. Entretanto, o presente artigo não teve poder para detectar evidências dessa associação.

Inúmeros carcinógenos, confirmados ou de grande potencial, fazem parte da rotina de atividades militares, como solventes orgânicos e inorgânicos, óleos, metais, fumaças, radiação ionizante e não-ionizante. Os bombeiros também estão potencialmente expostos a várias misturas particuladas; gases; metais como chumbo, cádmio, urânio, e substâncias químicas como hidrocarbonetos poliaromáticos, benzeno, formaldeídos, entre outras ${ }^{22}$.

LeMasters et al. ${ }^{22}$ publicaram uma metanálise sobre o risco de câncer entre bombeiros. Incluíram em sua pesquisa 32 estudos, divulgados entre 1966 e 2003, e encontraram uma estimativa sumária do risco de tumores cerebrais de 1,32 (IC95\% 1,12-1,54). Krishnan et al. ${ }^{10}$ coordenaram um estudo 
Tabela 3. Odds ratio bruta e ajustada do período de ocupações de acordo com a International Standard Classification of Occupations em anos para neoplasias intracranianas $(n=239)$, gliomas $(n=66)$ e meningiomas $(n=85)$ no Rio de Janeiro entre 1998 e 2002

\begin{tabular}{|c|c|c|c|c|c|c|}
\hline \multirow[b]{2}{*}{$\begin{array}{l}\text { ISCO - Tempo de ocupação } \\
\text { (anos) }\end{array}$} & \multicolumn{2}{|c|}{ Neoplasias intracranianas } & \multicolumn{2}{|c|}{ Gliomas } & \multicolumn{2}{|c|}{ Meningiomas } \\
\hline & $\begin{array}{l}\text { OR bruta } \\
(\text { IC } 95 \%)\end{array}$ & $\begin{array}{l}\text { OR ajustada* } \\
\quad \text { (IC95\%) }\end{array}$ & $\begin{array}{l}\text { OR bruta } \\
(\text { IC } 95 \%)\end{array}$ & $\begin{array}{l}\text { OR ajustada* } \\
\quad(\text { IC } 95 \%)\end{array}$ & $\begin{array}{l}\text { OR bruta } \\
\text { (IC95\%) }\end{array}$ & $\begin{array}{l}\text { OR ajustada }{ }^{*} \\
\quad(\text { IC95\%) }\end{array}$ \\
\hline \multicolumn{7}{|l|}{$\begin{array}{l}\text { ISCO } 1 \text { - Profissionais } \\
\text { técnicos }\end{array}$} \\
\hline 0 & 1,00 & 1,00 & 1,00 & 1,00 & 1,00 & 1,00 \\
\hline $1-5$ & $0,85(0,38-1,88)$ & $0,86(0,39-1,89)$ & $1,20(0,42-3,38)$ & $1,33(0,44-4,08)$ & $1,12(0,42-2,94)$ & $1,10(0,38-3,16)$ \\
\hline$\geq 6$ & $0,99(0,48-2,05)$ & $0,96(0,47-2,04)$ & $0,90(0,29-2,78)$ & $0,97(0,29-3,22)$ & $1,00(0,38-2,60)$ & $0,88(0,31-2,49)$ \\
\hline $\mathrm{p}$ de tendência & & 0,7863 & & 0,8825 & & 0,9771 \\
\hline \multicolumn{7}{|l|}{$\begin{array}{l}\text { ISCO } 3 \text { - Empregados de } \\
\text { escritório }\end{array}$} \\
\hline 0 & 1,00 & 1,00 & 1,00 & 1,00 & 1,00 & 1,00 \\
\hline $1-5$ & $0,86(0,45-1,62)$ & $0,88(0,48-1,64)$ & $0,78(0,29-2,11)$ & $0,89(0,32-2,50)$ & $0,68(0,27-1,72)$ & $1,12(0,41-3,03)$ \\
\hline$\geq 6$ & $1,34(0,76-2,36)$ & $1,35(0,97-2,38)$ & $2,01(0,95-4,25)$ & $2,33(1,02-5,29)$ & $1,43(0,70-2,90)$ & $2,11(0,92-4,83)$ \\
\hline $\mathrm{p}$ de tendência & & 0,4509 & & 0,1576 & & 0,5822 \\
\hline
\end{tabular}

ISCO 4 - Trabalhadores com vendas

0

$1-5$

$\geq 6$

$\mathrm{p}$ de tendência

ISCO 5 - Auxiliar de

serviços

\begin{tabular}{|c|c|c|c|c|c|c|}
\hline 0 & 1,00 & 1,00 & 1,00 & 1,00 & 1,00 & 1,00 \\
\hline $1-5$ & $0,61(0,36-1,04)$ & $0,65(0,39-1,08)$ & $0,56(0,24-1,32)$ & $0,58(0,24-1,38)$ & $1,16(0,59-2,28)$ & $1,21(0,59-2,44)$ \\
\hline$\geq 6$ & $1,04(0,69-1,56)$ & $1,11(0,73-1,69)$ & $1,39(0,77-2,51)$ & $1,47(0,78-2,77)$ & $1,41(0,81-2,45)$ & $1,07(0,57-2,00)$ \\
\hline $\mathrm{p}$ de tendência & & 0,8784 & & 0,2730 & & 0,2568 \\
\hline \multicolumn{7}{|c|}{ ISCO 6 - Agricultura } \\
\hline 0 & 1,00 & 1,00 & 1,00 & 1,00 & 1,00 & 1,00 \\
\hline $1-5$ & $0,75(0,26-2,13)$ & $0,72(0,27-1,90)$ & $2,02(0,68-6,06)$ & $1,95(0,64-5,96)$ & $1,20(0,37-3,89)$ & $1,33(0,39-4,54)$ \\
\hline$\geq 6$ & $2,57(1,17-5,74)$ & $2,44(1,14-5,18)$ & $2,43(0,86-6,85)$ & $2,32(0,80-6,72)$ & $2,11(0,79-5,63)$ & $2,08(0,75-5,88)$ \\
\hline $\mathrm{p}$ de tendência & & 0,0302 & & 0,0591 & & 0,1754 \\
\hline \multicolumn{7}{|c|}{$\begin{array}{l}\text { ISCO } 7 \text { - Transporte e } \\
\text { produção de equipamentos }\end{array}$} \\
\hline 0 & 1,00 & 1,00 & 1,00 & 1,00 & 1,00 & 1,00 \\
\hline $1-5$ & $1,18(0,70-2,00)$ & $1,20(0,73-1,97)$ & $1,25(0,61-2,59)$ & $1,24(0,60-2,57)$ & $1,30(0,61-2,77)$ & $1,59(0,72-3,51)$ \\
\hline$\geq 6$ & $1,18(0,79-1,79)$ & $1,12(0,72-1,74)$ & $0,95(0,51-1,77)$ & $0,89(0,45-1,76)$ & $0,91(0,45-1,84)$ & $1,22(0,59-2,56)$ \\
\hline $\mathrm{p}$ de tendência & & 0,4053 & & 0,8729 & & 0,2032 \\
\hline \multicolumn{7}{|c|}{ ISCO 9 - Forças Armadas } \\
\hline 0 & 1,00 & 1,00 & 1,00 & 1,00 & 1,00 & 1,00 \\
\hline $1-5$ & $1,14(0,47-2,77)$ & $1,13(0,48-2,66)$ & $1,54(0,47-5,00)$ & $1,72(0,49-6,03)$ & $0,51(0,11-2,32)$ & $0,97(0,20-4,80)$ \\
\hline$\geq 6$ & $2,85(0,49-21,40)$ & $2,61(0,49-13,92)$ & $4,23(0,58-30,66)$ & $5,03(0,64-39,27)$ & & \\
\hline $\mathrm{p}$ de tendência & & 0,3127 & & 0,1745 & & 0,1481 \\
\hline \multicolumn{7}{|c|}{ Outros (ISCO 2 e 8 ) } \\
\hline 0 & 1,00 & 1,00 & 1,00 & 1,00 & 1,00 & 1,00 \\
\hline $1-5$ & $1,12(0,23-5,36)$ & $1,06(0,26-4,39)$ & $1,00(0,11-9,13)$ & $1,02(0,11-9,55)$ & $0,78(0,09-7,05)$ & $0,78(0,08-7,52)$ \\
\hline$\geq 6$ & $0,56(0,02-7,87)$ & $0,49(0,04-5,57)$ & & & & - \\
\hline $\mathrm{p}$ de tendência & & 0,6151 & & 0,3680 & & 0,2758 \\
\hline
\end{tabular}

${ }^{*}$ OR ajustada por sexo, faixa etária e escolaridade

ISCO: International Standard Classification of Occupations; OR: Odds ratio; IC95\%: Intervalo de confiança de 95\% 
caso-controle na Baía de São Francisco, entre 1991 e 1999, com a finalidade de estimar a chance de gliomas em 1.129 casos de 56 categorias ocupacionais e encontraram OR de 6,31 (IC95\% 0,73-54,40) para bombeiros com muitos anos na profissão.

A associação de trabalhadores domésticos com tumores do SNC já foi mencionada por alguns estudos ${ }^{10,18}$. Porteiros, domésticas e outros trabalhadores da área podem ser expostos a diversas substâncias químicas presentes na composição de produtos de limpeza, inclusive solventes. Esses trabalhadores encontram-se agrupados no ramo de atividades econômicas Empregados domésticos (NACE 16), que inclui diversas ocupações listadas no grupo Auxiliar de serviços (ISCO 5). $\mathrm{Na}$ presente investigação, as razões de chance nos estratos com maior tempo de atividade foram positivas, mas estatisticamente não significativas, embora tenha sido detectada tendência de aumento do risco de meningioma com os anos de trabalho para esta atividade econômica.

O setor econômico Atacadista (NACE 7) compreende diversos perfis de venda a varejo e atacado, além de alguns serviços de reparo e manutenção de utensílios domésticos, vestuário e automotivo. Estudos relataram efeito protetor para o desenvolvimento de tumores do SNC nestes trabalhadores, sem apontar, contudo, hipóteses sobre tal achado ${ }^{10,11}$. Nesse estudo, verificou-se a presença de tendência estatisticamente significativa no efeito protetor para o desenvolvimento de gliomas em Trabalhadores com vendas (ISCO 4).

O grupo NACE 8, denominado Hotéis, contempla atividades em serviços hoteleiros, restaurantes, bares e cantinas. Alguns trabalhos apoiam a hipótese de que profissionais ligados ao ramo de preparo de alimentos estariam sob risco de desenvolver neoplasias cerebrais, pois são sujeitos à transmissão de agentes infecciosos provenientes de animais, como salmonela, listeria e brucelose, entre outros ${ }^{10,17,19}$. Nesta investigação foi observada tendência de efeito protetor para o desenvolvimento de meningiomas em trabalhadores dessa atividade econômica, a qual não é consoante com a literatura.

O ramo de atividade econômica Construção (NACE 6) é composto, neste estudo, por profissionais da área da construção civil que, majoritariamente, eram pedreiros. Poucas pesquisas foram realizadas explorando o risco de tumores cerebrais nesse grupo ocupacional, tendo relatado associação positiva $^{12}$, sem significância estatística, ou de proteção ${ }^{23}$ para o subtipo gliomas, ambas limítrofes. Na presente investigação, foi observada a presença de tendência de efeito protetor para o desenvolvimento de meningiomas nesse ramo de atividade.

Os profissionais da área de saúde (NACE 14) com 6 anos ou mais de tempo de trabalho mostraram OD de 3,5 para meningiomas. Alguns autores ${ }^{10,23}$ já apontaram associação de médicos, enfermeiros, dentistas e demais profissionais da área de saúde com as neoplasias intracranianas, em virtude de potenciais exposições a agentes químicos e físicos, como desinfetantes, antissépticos, quimioterápicos, radiação, além dos biológicos no contato com pacientes enfermos, cirurgias, análise de materiais por patologistas. Ademais, estes trabalhadores teriam como característica um acesso maior aos serviços de saúde, o que resultaria na maior probabilidade de diagnóstico destas neoplasias.

O presente estudo caso-controle não foi desenvolvido, especificamente, para a abordagem ocupacional, e sim para explorar diversas exposições de cunho pessoal e ambiental. $\mathrm{O}$ aprofundamento da análise ocupacional surgiu após emergirem algumas hipóteses da primeira avaliação realizada por Monteiro ${ }^{14}$. Assim, foram analisados os ramos de atividade econômica e as ocupações dos sujeitos, e não as exposições do ambiente de trabalho, em particular. A metodologia de classificação ocupacional em padrões é considerada válida para posterior inferência sobre exposições, porém não possibilita precisar sua variabilidade dentro de um mesmo grupo ocupacional ${ }^{24}$.

Este trabalho apresenta algumas limitações, também encontradas em outros estudos caso-controle de neoplasias intracranianas: pequeno tamanho de amostra; acurácia da resposta dos sujeitos com doença neurológica, e de familiares quando estes se encontram impossibilitados de responder; e potencial viés de memória. A observação de eventuais diferenças entre os resultados encontrados na literatura, magnitude e direção da associação podem assim ser devido às restrições apontadas.

Os resultados deste estudo apresentaram, majoritariamente, um amplo intervalo de confiança que se deve, essencialmente, ao reduzido número de indivíduos expostos. Além disso, ao se proceder a estratificações, como tipo histológico, houve perda no poder do estudo.

Por outro lado, são pontos fortes deste artigo: a avaliação por subtipo histológico de neoplasias intracranianas (gliomas e meningiomas); a alta porcentagem (80,0\%) de confirmação histopatológica dos casos, além da forte concordância (acima de 80,0\%) na segunda leitura histopatológica da lâmina; o número reduzido de respondentes substitutos $(15,1 \%)$ em comparação à média de 43 a $46 \%$ de outros estudos ${ }^{10,23}$, bem como elevado percentual $(94,4 \%)$ de participação dos sujeitos elegíveis. Além disso, são poucos os estudos brasileiros que se propuseram a estimar associações entre ocupações e neoplasias intracranianas.

Os resultados deste trabalho, portanto, sugerem que tanto ramos de atividade econômica quanto grupos ocupacionais específicos podem apresentar potencial risco para o desenvolvimento de neoplasias intracranianas. A análise por gliomas 
e meningiomas exacerbou algumas associações, o que pode indicar fatores etiológicos ocupacionais diferenciados. Sendo assim, este campo de conhecimento carece de mais estudos que visem à identificação de exposições específicas dentro dos ambientes de trabalho.

\section{AGRADECIMENTOS}

À pesquisadora Rosalina Jorge Koifman pela valiosa contribuição na classificação das ocupações e análises deste trabalho e à Coordenação de Aperfeiçoamento de Pessoal de Nível Superior (CAPES), que financiou a bolsa de Mestrado de um dos autores.

\section{REFERÊNCIAS}

1. Wrensch M, Minn Y, Chew T, Bondy M, Berger MS. Epidemiology of primary brain tumors: current concepts and review of the literature. Neuro Oncol. 2002;4(4):278-99.

2. World Health Organization. International Agency for Research on Cancer. Globocan 2008 - estimated cancer incidence, mortality, prevalence and disability-adjusted life years (DALYs) Worldwide in 2008 [Internet]. Available from: http://globocan.iarc.fr

3. Curado MP, Edwards B, Shin HR, Storm H, Ferlay J, Heanue M, Boyle P. Cancer incidence in five continents, Vol. IX. Lyon: IARC Scientific Publications; 2007.

4. Monteiro GTR, Koifman S. Mortalidade por tumores de cérebro no Brasil, 1980-1998. Cad Saúde Pública. 2003;19(4):1139-51.

5. Wünsch-Filho V, Koifman S. Tumores malignos relacionados ao trabalho. In: Mendes R. Patologia do trabalho. Rio de Janeiro: Atheneu; 2003. p. 989-1040.

6. Khuder SA, Mutgi AB, Schaub EA. Meta-analyses of brain cancer and farming. Am J Ind Med. 1998;34(3):252-60.

7. Miranda-Filho AL, Monteiro GT, Meyer A. Brain cancer mortality among farm workers of the State of Rio de Janeiro, Brazil: a population-based case-control study, 1996-2005. Int J Hyg Environ Health. 2012;215(5):496-501.

8. Alder N, Fenty J, Warren F, Sutton AJ, Rushton L, Jones DR, Abrams KR. Meta-analysis of mortality and cancer incidence among workers in the synthetic rubber-producing industry. Am J Epidemiol. 2006;164(5):405-20.

9. Lewis RJ, Schnatter AR, Drummond I, Murray N, Thompson FS, Katz AM, Jorgensen G, Nicolich MJ, Dahlman D, Thériault G. Mortality and cancer morbidity in a cohort of Canadian petroleum workers. Occup Environ Med. 2003;60(12):918-28.

10. Krishnan G, Felini M, Carozza SE, Miike R, Chew T, Wrensch M. Occupation and adult gliomas in the San Francisco Bay Area. J Occup Environ Med. 2003;45(6):639-47.

11. Navas-Acién A, Pollán M, Gustavsson P, Plato N. Occupation, exposure to chemicals and risk of gliomas and meningiomas in Sweden. Am J Ind Med. 2002;42(3):214-27.

12. Pan SY, Ugnat AM, Mao Y. Occupational risk factors for brain cancer in Canada. J Occup Environ Med. 2005;47(7):704-17.

13. Berg G, Spallek J, Schüz J, Schlehofer B, Böhler E, Schlaefer K, Hettinger I, Kunna-Grass K, Wahrendorf J, Blettner M. Occupational exposure to radio frequency/microwave radioation and the risk of brain tumors: Interphone Study Group, Germany. Am J Epidemiol. 2006;164(6):538-48.

14. Monteiro GTR. Fatores ambientais associados às neoplasias intracranianas: estudo caso-controle na região metropolitana do Rio de Janeiro [tese]. Rio de Janeiro (RJ): Escola Nacional de Saúde Pública Sérgio Arouca. FIOCRUZ; 2004.

15. Nomenclature générale des Activités économiques dans les Communautés Européennes. Statistical Classification of Economic Activities in the European Community. Luxembourg: Eurostat, 1996.

16. International Labour Office (ILO). International Standard Classification of Occupations (ISCO). Geneva: ILO, 1981

17. Schlehofer B, Hettinger I, Ryan P, Blettner M, Preston-Martin S, Little J, Arslan A, Ahlbom A, Giles GG, Howe GR, Ménégoz F, Rodvall Y, Choi WN, Wahrendorf J. Occupational risks factors for low grade and high grade glioma: results from an internacional case control study of adult Brain tumours. Int J Cancer. 2005;113:116-25.

18. Preston-Martin S, Mack W, Henderson BE. Risk factors for gliomas and meningiomas in males in Los Angeles County. Cancer Res. 1989;49(21):6137-43.

19. Ménégoz F, Little J, Colonna M, Arslan A, Preston-Martin S, Schlehofer B, Blettner M, Howe GR, Ryan P, Giles GG, Rodvall Y, Choi WN. Contacts with animals and humans as risk factors for adult brain tumours. An international case-control study. Eur J Cancer. 2002;38(5):696-704.

20. Archer GE, Sampson JH, Bigner DD. Viruses and oncogenes in brain tumors. J Neurovirol. 1997;3(Suppl 1):76-7.

21. Silva M, Santana VS, Loomis D. Mortalidade por câncer em militares da Marinha do Brasil. Rev Saúde Pública. 2000;34(4):373-9.

22. LeMasters GK, Genaidy AM, Succop P, Deddens J, Sobeih T, BarrieraViruet H, Dunning K, Lockey J. Cancer risk among firefighters: a review and meta-analysis of 32 studies. J Occup Environ Med. 2006;48(11):1189-202.

23. De Roos AJ, Stewart PA, Linet MS, Heineman EF, Dosemeci M, Wilcosky T, Shapiro WR, Selker RG, Fine HA, Black PM, Inskip PD. Occupation and the risk of adult glioma in the United States. Cancer Causes Control. 2003;14(2):139-50.

24. Fritschi L, Siemiatycki J, Richardson L. Self-assessed versus expertassessed occupational exposures. Am J Epidemiol, 1996;144(5):521-7.

Recebido em: 20/03/2012 Aprovado em: 20/06/2012 